ACCEPTED MANUSCRIPT

\title{
Magneto-dependent stress relaxation of magnetorheological gels
}

To cite this article before publication: Yangguang Xu et al 2017 Smart Mater. Struct. in press https://doi.org/10.1088/1361-665X/aa89ac

\section{Manuscript version: Accepted Manuscript}

Accepted Manuscript is "the version of the article accepted for publication including all changes made as a result of the peer review process, and which may also include the addition to the article by IOP Publishing of a header, an article ID, a cover sheet and/or an 'Accepted Manuscript' watermark, but excluding any other editing, typesetting or other changes made by IOP Publishing and/or its licensors"

This Accepted Manuscript is @ 2017 IOP Publishing Ltd.

During the embargo period (the 12 month period from the publication of the Version of Record of this article), the Accepted Manuscript is fully protected by copyright and cannot be reused or reposted elsewhere.

As the Version of Record of this article is going to be / has been published on a subscription basis, this Accepted Manuscript is available for reuse under a CC BY-NC-ND 3.0 licence after the 12 month embargo period.

After the embargo period, everyone is permitted to use copy and redistribute this article for non-commercial purposes only, provided that they adhere to all the terms of the licence https://creativecommons.org/licences/by-nc-nd/3.0

Although reasonable endeavours have been taken to obtain all necessary permissions from third parties to include their copyrighted content within this article, their full citation and copyright line may not be present in this Accepted Manuscript version. Before using any content from this article, please refer to the Version of Record on IOPscience once published for full citation and copyright details, as permissions will likely be required. All third party content is fully copyright protected, unless specifically stated otherwise in the figure caption in the Version of Record.

View the article online for updates and enhancements. 


\title{
Magneto-dependent stress relaxation of magnetorheological gels
}

\author{
Yangguang Xu $u^{a, b} \psi$, Taixiang Liu ${ }^{c}$, Guojiang Liao ${ }^{b}$ and Gilles Lubineau ${ }^{a}{ }^{\dagger}$
}

${ }^{a}$ King Abdullah University of Science and Technology (KAUST), Physical Science and Engineering Division, COHMAS Laboratory, Thuwal 23955-6900, Saudi Arabia

${ }^{b}$ Institute of Systems Engineering, China Academy of Engineering Physics (CAEP), Mianyang 621999, China

${ }^{c}$ Research Center of Laser Fusion, China Academy of Engineering Physics (CAEP), Mianyang, 621900, China

†Corresponding Author:

Yangguang Xu:

E-mail: yangguang.xu@kaust.edu.sa

Gilles Lubineau:

E-mail: gilles.lubineau@kaust.edu.sa 


\begin{abstract}
The stress relaxation behaviors of magnetorheological (MR) gels under stepwise shear loading are systematically investigated. The particle-enhanced effect, the magneto-induced effect, and the temperature-enhanced effect on the stress relaxation of MR gels are discussed. For further analysis of the magneto-induced stress relaxation mechanism in MR gels, a phenomenological model is established to describe the stress relaxation behavior of the matrix and the magnetic particle chains. All characteristic parameters introduced in the model, i.e. relaxation time, instantaneous modulus, and stable modulus, have well-defined physical meanings and are fitted based on the experimental results. The influence of each parameter on the macroscopic response is discussed and it is found that the relaxation stress induced by the magnetomechanical coupling effect plays an important role in the stress relaxation process of MR gels .
\end{abstract}

\title{
Key words
}

magnetorheological gels; stress relaxation; magneto-induced effect; temperature-enhanced effect 


\section{1}

\section{Introduction}

Magnetorheological (MR) gels are a kind of magneto-sensitive smart material prepared by dispersing micrometer-sized magnetic particles into a polymer matrix without sufficient crosslinking [1-6]. Due to the weak polymer matrix, the magnetic particles will rearrange to form chainlike structures aligned parallel to the direction of magnetic field. After the external magnetic field is removed, the structured particle chains remain, which means that the particle sedimentation problem (existing in MR fluids) can be solved to some extent in an MR gel (MRG). The magnetoinduced evolution of the microstructure changes the rheological properties of the MRG remarkably [7-9], which indicates that the rheological properties of MRGs may be controlled by magnetic fields. In turn, changes to the rheological properties in the presence of a magnetic field can be used to monitor and understand the evolution of the microstructure.

The polymer matrix of MRGs shows typical viscoelastic behaviors, resulting in more complex rheological behaviors than seen in conventional MR fluids and MR elastomers. At the same time, the magnetic fillers changes the viscoelastic properties of MRGs in the presence of an external magnetic field. Therefore, this mechanical-magnetic coupling behavior is the key point for investigating the MR mechanism, and the characterization of viscoelasticity in the presence of a magnetic field is the basis for describing the magneto-mechanical coupling behavior of MRGs. The time-dependent rheological properties actuated by different external loading conditions, such as creep, stress relaxation, or oscillatory loading, are important for many approaches to characterizing viscoelasticity. Among them, dynamic mechanical analysis is the most popular method to investigate the viscoelastic behaviors of MR gels $[10,11]$. Indeed, the mechanism of microstructure evolution in MRGs can be studied in depth by analyzing the magneto-sensitivity of the storage modulus and loss factor. However, understanding the magneto-induced viscoelasticity only from the dynamic mechanical property is not comprehensive, and transient experiments under stepwise loading are also needed. Normally, the response of MRGs is also strain rate dependent. Dynamic mechanical experiments investigate the behavior in high frequency regime (i.e. short time period) while transient experiments probe the rate dependency of mechanical properties at a larger time scale because it covers all the possible loading frequency range [12, 13]. An et al. suggested that using a stress-relaxation modulús calculated from dynamic oscillation data to analyze the phase transition of MR materials is a beneficial method to analyze viscoelasticity by combing both the dynamic and transient properties [14].

The simplest transient experiment consists of applying a constant loading to the sample and recording its time-dependent response. If the stress is kept constant (the strain varying with time), the test is defined as creep. Inversely, if the strain is kept constant (the stress varying with time), the test is defined as stress relaxation [12]. Creep and stress relaxation tests are the foundation of 
investigating the response of the polymer to arbitrary actuating loadings. The relationship between microstructure and rheology is easier to be interpreted through stress relaxation than creep. Furthermore, the relaxation function, i.e. relaxation modulus, is more intuitive and more easily understood than the creep compliance [15]. The stress relaxation of MR fluids was investigated by Li et al. They found that there exists an obvious linear viscoelastic range and that the stable relaxation modulus is sensitive to a magnetic field [16]. A similar result was also found in MR elastomers [17]. As for MR elastomers, the magnetic particles are restricted by a rubber matrix. The viscous fluid in MR fluids, on the other hand, hardly constrains the magnetic particles at all, so that the magnetic interaction between the magnetic particles in the presence of a magnetic field determines the stress relaxation behavior of MR fluids. These mechanisms indicate that the elasticity is the main deformation form in stress relaxation for MR fluids and MR elastomers, which can be confirmed from the abovementioned experimental results. A MRG is an intermediate material system between a MR fluid and a MR elastomer; the constraining effect of a polymer matrix on the magnetic particles is weaker than that of an elastic rubber but stronger than that of a viscous fluid. The stress relaxation of MRGs is determined by the polymer matrix as well as the magnetic particles.

However, there are few published works/about the stress relaxation behavior of MRGs. To further understand the magneto-mechanical coupling mechanism and to provide comprehensive experimental data for theoretical analysis and engineering applications, the stress relaxation behavior of MRGs under shear loading is systematically investigated in this paper. We first test the stress relaxation of MRGs actuated by different strains. Then, we investigate the influences of particle distribution, particle content, and magnetic field strength on the stress relaxation behavior of MRGs. Finally, we discuss the temperature-enhanced effect of MRGs on the stress relaxation process. At the same time, we establish a phenomenological model that can approximately describe the magneto-dependent stress relaxation behavior of MRGs. Based on the experimental results, we describe the relationship between the characteristic parameters in the model (i.e. relaxation time, instantaneous modulus, and stable modulus) and the experimental variables (particle, magnetic field, and temperature), thereby enhancing our understanding of the magneto-mechanical coupling mechanism in MRGs.

\section{Experimental}

\subsection{Fabrication of MRGs}

The polymer matrix of the MRGs was synthesized by following the procedure detailed in our previous work [18]. Toluene diisocyanate (TDI, 2,4 80\%, 2,6 20\%, Tokyo Chemical Industry Co., Ltd, Japan) and polypropylene glycol (PPG-1000, Mn =1000, Sigma-Aldrich (Shanghai) Trading Co., Ltd, China) were the main reactants. Dipropylene glycol (Sigma-Aldrich (Shanghai) Trading 
Co., Ltd, China) and 1-methyl-2-pyrrolidone (Sinopharm Chemical Reagent Co., Ltd, China) were chosen as the chain extender and the thinner, respectively. The weight ratio of thinner to polymer matrix was set to 0.1 to ensure solid-like MRGs can be obtained. The carbonyl iron particles (type $\mathrm{CN}$, provided by BASF in Germany, with an average radius of $6 \mu \mathrm{m}$ ) were dispersed evenly throughout the polymer matrix with weight fractions of $40 \mathrm{wt} \%, 50 \mathrm{wt} \%, 60 \mathrm{wt} \%$, and $70 \mathrm{wt} \%$. The plasticine-like MRG samples were finally obtained by keeping the mixture in an oven at $40{ }^{\circ} \mathrm{C}$ for 12 hours. The samples were named MRG-40, MRG-50, MRG-60, and MRG-70, corresponding to their respective weight fraction of magnetic particles.

\subsection{Experimental procedure}

A commercial parallel-plate rheometer (Physica MCR 301, Anton Paar Co., Austria) was used to carry out the stress relaxation experiment on the MRGs. A stress relaxation measurement module was built in the RHEOPLUS software, from which the experimental conditions, such as strain amplitude, relaxation time, and temperature, were easily set. Moreover, the magnetic field, applied perpendicularly through the sample, was adjusted by changing the current value in the magnetocontrollable accessory, the MRD 180. Due to the plastic feature of our sample [10], the initial sample that was placed between the plates was spherical. The gap size of the plates was then adjusted to $1 \mathrm{~mm}$ and the sample was squeezed to $1 \mathrm{~mm}$. To ensure uniformity of different samples, we simply needed to keep the volume of the samples the same. Finally, the diameter of the MRG sample between the parallel plates was $20 \mathrm{~mm}$ and the thickness was $1 \mathrm{~mm}$. In our pre-experiment, it was found that the relaxation stress was stable when the relaxation time was set to 300 seconds, so the relaxation time in all our experiments was set to 300 seconds. The influences of strain amplitude, magnetic field, and temperature on the stress relaxation of the MRGs will be discussed individually.

\section{Stress relaxation model}

It is difficult to construct a constitutive model that can predict the macroscopic mechanical behaviors of polymer due to its complex molecular motion. The spring-dashpot model is an effective tool for describing the linear viscoelastic behavior of polymers in a phenomenological manner [19-22]. Indeed, the stréss relaxation behavior of most polymers can be described by the standard linear solid model with three characteristic parameters (the stiffness of two springs and the viscosity of one dashpot, as the right part of Fig. 1b shown). However, an MRG is a polymer matrix containing magnetic particle fillers. The interaction between adjacent magnetic particles in the same particle chain should also be considered. We describe here the magneto-induced mechanical response of particle chains under shear loading using the classic magnetic dipole theory. 
(a)
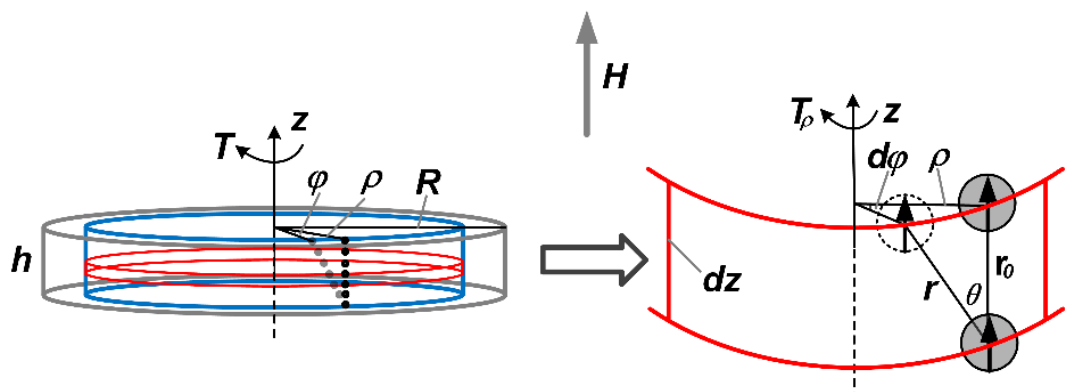

(b)

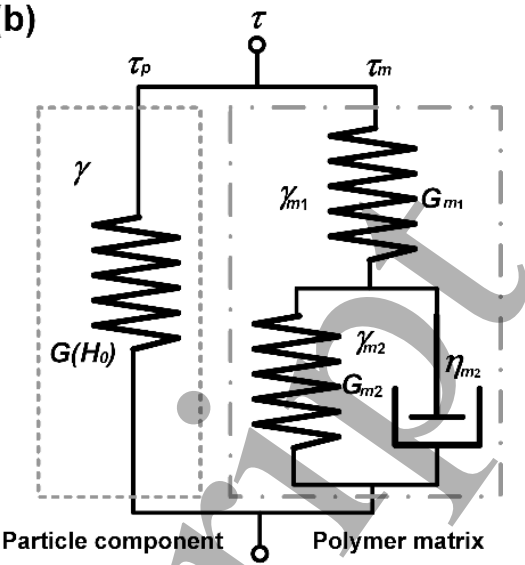

Figure 1 A schematic of the magnetic dipole model of a single particle chain under rotating shear loading (a) and the stress relaxation model of a MRG (b).

Figure 1a shows a classic sample (outer radius: R) under shear loading. A differential element with an arbitrary radius $\rho(0 \leq \rho \leq \mathrm{R})$ is also introduced. Each particle is assumed to be a magnetic dipole and magnetized evenly (we assume that the diameter and the magnetic dipole moment are the same for all the particles). The interaction energy between two magnetic dipoles under an external magnetic field $H_{0}$ is:

$$
E_{12}=\frac{1}{4 \pi \mu_{0} \mu_{r}} \frac{m^{2}\left(1-3 \cos ^{2} \theta\right)}{|r|^{3}},
$$

where $\mu_{0}$ is the permeability of the vacuum, $\mu_{r}$ is the relative permeability of the matrix, $m$ is the magnetic dipole moment of the particle filler, $\theta$ is the orientation of the dipole with respect to the external magnetic field (i.e. the intersection between $r$ and $r_{0}$ ), and $r$ is the distance between two particles after deformation.

The magnetic dipole moment $m$ follows Eq. (2), where $a$ is the radius of a particle, $\chi$ is the susceptibility of the particle filler, and $H$ is the magnetic field strength at the position of the considered particle:

$$
m=\frac{4}{3} \pi a^{3} \mu_{0} \mu_{r} \chi H
$$

We follow here a similar construction as Shen et al [23]. The magnetic field at particle $i$ consists of the external magnetic field $H_{0}$ and the magnetic field $H_{j}$ generated by the other particles in the same particle chain except particle $i$ : we suppose that two adjacent particles in the same particle chain have the same distance. Doing so, the magnetic field $H_{i}$ at particle $i$ can be expressed as:

$$
H_{i}=H_{0}+\sum_{j \neq i}^{n} H_{j}=H_{0}+2 \sum_{j=1, j \neq i}^{n} \frac{m\left(3 \cos ^{2} \theta-1\right)}{4 \pi \mu_{0} \mu_{r}(j r)^{3}} .
$$

Therefore, we can obtain the magnetic dipole moment of particle $i$ by substituting Eq. (3) into Eq. 
(2):

$$
m_{i}=\frac{4}{3} \pi a^{3} \mu_{0} \mu_{r} \chi\left[H_{0}+2 \sum_{j=1, j \neq i}^{n} \frac{m_{j}\left(3 \cos ^{2} \theta-1\right)}{4 \pi \mu_{0} \mu_{r}(j r)^{3}}\right] .
$$

If the particle chain is long enough, which means that $n$ in Eq. (4) is a large number, then the $m$ of each particle in this chain can be assumed to have the same value. That is:

$$
m_{i}=m_{j}=m \text {. }
$$

Assuming small perturbations, we can consider that $r \approx r_{0}$ and $\theta \approx 0$, and so Eq. (4), can be simplified as:

$$
m=\frac{4 \pi a^{3} \mu_{0} \mu_{r} \chi H_{0}}{3-4 C \chi\left(a / r_{0}\right)^{3}}, C=\sum_{j=1}^{n} \frac{1}{j^{3}} .
$$

Finally, the magnetic interaction energy of particle $i$ is:

$$
\begin{gathered}
E_{i}=\sum_{j \neq i} E_{i j}=2 \sum_{j=1, j \neq i}^{n} E_{i j}=\frac{C m^{2}\left(1-3 \cos ^{2} \theta\right)}{2 \pi \mu_{0} \mu_{r} r^{3}}, \\
\cos ^{2} \theta=\frac{r_{0}^{2}}{r^{2}} \approx \frac{r_{0}^{2}}{r_{0}^{2}+(\rho d \varphi)^{2}}
\end{gathered}
$$

If we define $\gamma_{\rho}=\frac{\rho d \varphi}{r_{0}}=\frac{\rho d \varphi}{d z}$, then $E_{i}$ can be expressed as:

$$
E_{i}=\frac{C m^{2}\left(1-\frac{3 r_{0}^{2}}{r_{0}^{2}+(\rho d \varphi)^{2}}\right)}{2 \pi \mu_{0} \mu_{r}\left[r_{0}^{2}+(\rho d \varphi)^{2}\right]^{3 / 2}}=\frac{C m^{2}\left(\gamma_{\rho}^{2}-2\right)}{2 \pi \mu_{0} \mu_{r} r_{0}^{3}\left(\gamma_{\rho}^{2}+1\right)^{5 / 2}} .
$$

The particle number in unit volume is:

$$
n=\frac{3 \phi}{4 \pi a^{3}},
$$

where $\phi$ is the volume fraction of particles for the whole sample, so that the energy of the unit volume around $\rho$ is:

$$
U=n E_{i}=\frac{3 \phi C m^{2}\left(\gamma_{\rho}^{2}-2\right)}{8 \pi^{2} \mu_{0} \mu_{r} a^{3} r_{0}^{3}\left(\gamma_{\rho}^{2}+1\right)^{5 / 2}} .
$$

The shear stress around $\rho$ can be worked out by differentiating the Eq. (11) as [24]

$$
\tau_{\rho}=\frac{\partial U}{\partial \gamma_{\rho}}=\frac{9 \phi C m^{2}\left(4-\gamma_{\rho}^{2}\right)}{8 \pi^{2} \mu_{0} \mu_{r} a^{3} r_{0}^{3}\left(1+\gamma_{\rho}^{2}\right)^{7 / 2}} \gamma_{\rho} \approx \frac{9 \phi C m^{2}}{2 \pi^{2} \mu_{0} \mu_{r} a^{3} r_{0}^{3}} \gamma_{\rho}=G_{\rho}\left(H_{0}\right) \gamma_{\rho} .
$$

The maximum error induced by the approximation in Eq. (12) will not exceed $0.04 \%$ if the shear strain is less than $1 \%$; this accuracy is acceptable for our analysis.

By integrating the whole cross section, we can obtain the moment of the sample at the center point of the sample as: 


$$
T=\int_{A} \rho \tau_{\rho} d A=\int_{A} \rho G_{\rho}\left(H_{0}\right) \rho \frac{d \varphi}{d z} d A=G_{\rho}\left(H_{0}\right) \frac{d \varphi}{d z} \int_{0}^{2 \pi} \int_{0}^{R} \rho^{3} d \rho d \theta=\frac{\pi R^{4}}{2} G_{\rho}\left(H_{0}\right) \frac{d \varphi}{d z} .
$$

The total shear stress $\tau$ measured by the rotational rheometer is related to the moment, and the total shear strain $\gamma$ is calculated based on the torsion angle $\varphi$, so the conversion formula according to the definition in the manual of the rheometer is:

$$
\tau=\frac{2 T}{\pi R^{3}}, \gamma=\frac{R \varphi}{10 h} .
$$

If we integrate the moment in the direction of the thickness, then the torsion angle of the surface of revolution (i.e. the top face in Figure 1a) can be worked out as:

$$
\varphi=\int_{0}^{h} \frac{2 T}{\pi R^{4} G_{\rho}\left(H_{0}\right)} d z=\frac{2 T h}{\pi R^{4} G_{\rho}\left(H_{0}\right)} .
$$

The relationship between shear stress and shear strain can finally be obtained based on Eq. (14) and Eq. (15) as:

$$
\tau=10 G_{\rho}\left(H_{0}\right) \gamma=\frac{45 \phi C m^{2}}{\pi^{2} \mu_{0} \mu_{r} a^{3} r_{0}^{3}} \gamma=G\left(H_{0}\right) \gamma,
$$

where $G\left(H_{0}\right)$ is the magneto-induced modulus of the MRG under rotational shear loading. Under a specific magnetic field, $G\left(H_{0}\right)$ is constant, so this part can be simplified as a spring.

With this realization, the constitutive relation can be described by the phenomenological model as shown in Fig. 1b, whose mathematical expression is:

$$
\begin{aligned}
& \tau=\tau_{p}+\tau_{m} \\
& \gamma=\gamma_{m 1}+\gamma_{m 2} \\
& \tau_{m}=G_{m 1} \cdot \gamma_{m 1}=G_{m 2} \cdot \gamma_{m 2}+\eta_{m 2} \cdot \dot{\gamma}_{m 2} . \\
& \tau_{p}=G\left(H_{0}\right) \cdot \gamma
\end{aligned}
$$

If $\gamma$ is a stepwise strain $\left(\gamma=\gamma_{0} \cdot H(t)\right.$, where $H(t)$ is a unit Heaviside function, i.e. $H(t)=0$ when $t<0$ and $H(t)=1$ when $t>0$, then the relaxation stress of the MRG is:

$$
\tau=\gamma_{0}\left(\frac{G_{m 1} G_{m 2}}{G_{m 1}+G_{m 2}}+G\left(H_{0}\right)\right)+\frac{\gamma_{0} G_{m 1}^{2}}{G_{m 1}+G_{m 2}} e^{-\frac{\left(G_{m 1}+G_{m 2}\right) t}{\eta_{m 2}}}
$$

(the detailed mathematical derivation of Eqs. (17) and (18) can be found in the Appendix). The modulus of the spring and the viscosity of the dashpot in Fig. 1b can be obtained by fitting the experimental results based on Eq. (18). Furthermore, some critical characteristic parameters can be calculated. Here, we define three characteristic parameters according to Eq. (18) as follows:

1) Relaxation time:

$$
t_{1}=\frac{\eta_{m 2}}{G_{m 1}+G_{m 2}}
$$

2) Instantaneous modulus when $\mathrm{t} \rightarrow 0$ : 


$$
G_{i n s}=G_{m 1}+G\left(H_{0}\right)
$$

3) Stable modulus when $t \rightarrow \infty$ :

$$
G_{s t a b}=\frac{G_{m 1} G_{m 2}}{G_{m 1}+G_{m 2}}+G\left(H_{0}\right)
$$

The magneto-mechanical coupling module between the particle and the matrix is not introduced into the stress relaxation model [21]. However, the magneto-mechanical coupling effect between the particle and the matrix has great influence on the rheological behaviors according to our previous report on magneto-induced creep and recovery behaviors of MRGs [26]. It is assumed that the rheological properties will not change in the absence of a magnetic field, if the microstructure distribution is fixed. When applying an external magnetic field, the increment of the modulus consists of both the stiffness of the particle chains and the mechanical-magnetic coupling effect. The stiffness of the particle chains is determined only by the magnetic field (see the left part of Figure 1b). The influence of the magneto-mechanical coupling effect on the rheological properties of MRGs can be reflected from the changes in the three parameters of the standard linear solid model (the right part of Figure 1b) after applying the magnetic field.

\section{Results and discussion}

\subsection{Determination of the linear viscoelastic (LVE) range}

There are many methods for determining the linear viscoelastic (LVE) range of materials. The shape of the stress-strain curves under a quasi-static loading condition is the simplest way [26, 27]. The Payne effect under an oscillatory loading condition and the shape of the hysteresis loop in an oscillating period can also be used to determine the LVE range [28-30]. Still, the Fourier rheology is needed for a more accurate analysis of the viscoelasticity [31]. Moreover, the transient response under stepwise loading could be used as a criterion to determine whether the material is linear viscoelastic or not: the relaxation modulus (or creep compliance) of a linear viscoelastic material is independent of the amplitude of a stepwise strain (or stepwise stress). Figure 2 shows a series of stress relaxation curves obtained by applying the corresponding stepwise strains with different amplitudes (Figure 2a) to MRG-70. The relaxation modulus $\left(G(t)=\tau(t) / \gamma_{0}\right)$ can be calculated directly from the stress relaxation curves (the inset of Figure $2 b$ ). Though the relaxation modulus under the $0.05 \%$ strain fluctuates sharply, possibly because the weak response signal is interfered by the noise signal, the relaxation moduli under different strains superpose well on each other, indicating that MRG-70 is linear viscoelastic within the selective range of strain amplitude. 

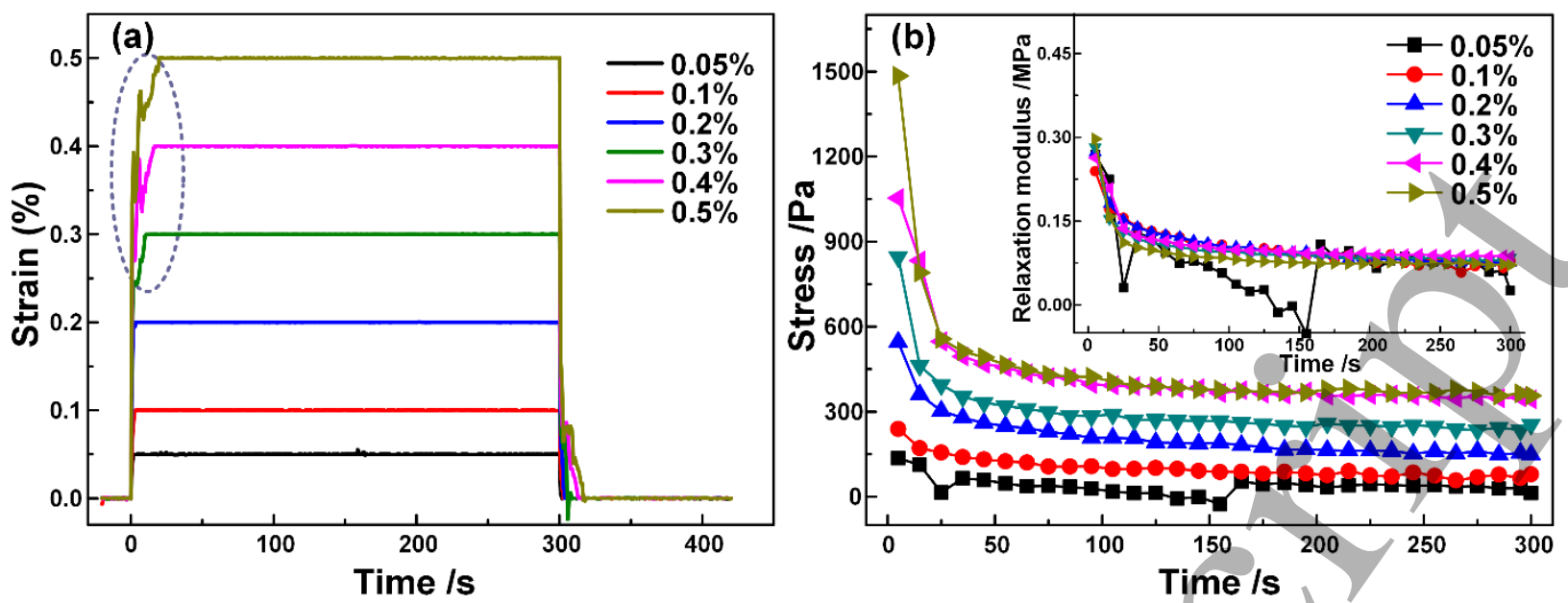

Figure 2 Strain-time curves (a) and stress relaxation curves (b) of MRG-70, actuated by different strains; the inset is the time dependence of the relaxation moduli. All of the experiments are carried out under a $95.6 \mathrm{mT}$ magnetic field.

It can be seen from the area indicated by a dashed ellipse in Figure 2 that the strain cannot increase to the equilibrium value immediately if the strain amplitude exceeds $0.3 \%$, a gradual increasing process of strain is needed (the time for the strain reach to the setting value increases with the increasing of strain amplitude: $10 \mathrm{~s}, 16 \mathrm{~s}$, and $19 \mathrm{~s}$ correspond to the strain amplitude of $0.3 \%, 0.4 \%$, and $0.5 \%$, respectively). The wall slipping between rotator and sample happens at large strain amplitude, so that the test data is not credible and the real stress relaxation of MRG cannot be reflected from the response stress. Therefore, $0.2 \%$ is chosen for the amplitude of the stepwise strain in our next measurements. With this strain amplitude condition, the sample can fall into the LVE range (this coincides with the result we obtained by the dynamic mechanical method [8]), and the wall slipping induced by large strain amplitude can be avoided as well.

\subsection{Particle-enhanced effect and the influence of particle distribution}

It is expected that the concentration of magnetic particle will significantly affect the dynamic properties [32, 33] and electrical conductivity [34, 35] of the MRG. The stress relaxation experiment also confirms the particle-enhanced effect. Figure 3 demonstrates that the stress relaxation curves of the MRG change with different particle contents: the instantaneous modulus and the stable modulus increase, with increasing particle concentration (Table 1). On the one hand, the polymer matrix is adsorbed by the particle fillers to form a covering gels (i.e. the gel covering closely to the surface of the particle), which improves the rheological properties of the MRG; on the other hand, the secondary network consisting of the particles will also enhance the rheological properties of MRG [36]. With increasing particle concentration, the density of the covering gel increases, and the secondary particle network becomes more complex; thus, the particle-enhanced effect becomes more obvious. 

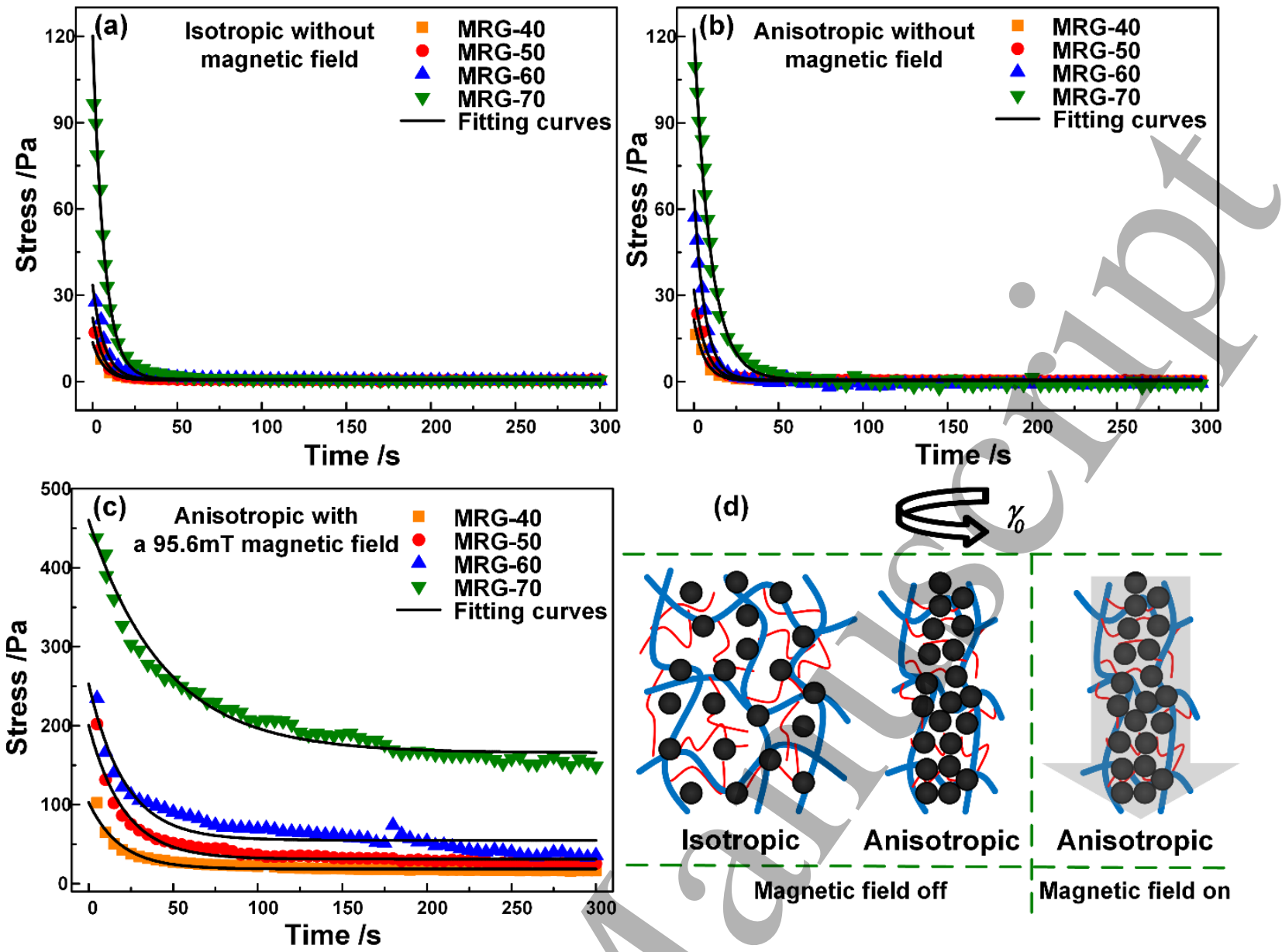

(d)

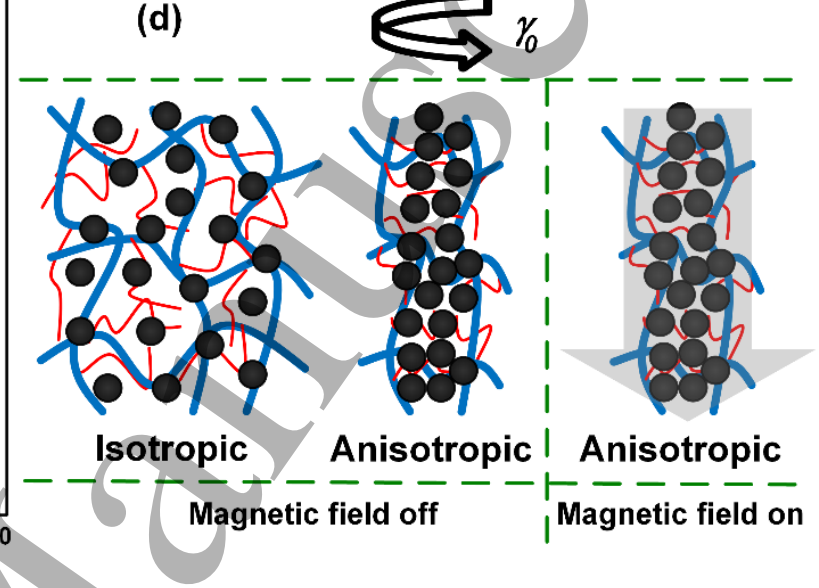

Figure 3 The stress relaxation behaviors of magnetorheological gels with different particle concentrations under different particle distribution states (isotropic without magnetic field (a), anisotropic without magnetic field (b), and anisotropic in the presence of magnetic field (c)) and a microstructure schematic of these different particle distribution states (d).

Figure $3 \mathrm{~d}$ illustrates three different distribution states of MRGs, which is the most remarkable distinction between an MRG and an MR fluid or an MR elastomer, which each possess only two particle distribution states. In the first state, like an MR fluid, in the absence of a magnetic field the magnetic particles maintain a randomly dispersed, isotropic state. In the second state, like an MR elastomer, the magnetic particles are firmly restricted by the surrounding rubber matrix and the particle distribution will maintain its initial isotropic or anisotropic state, whether a magnetic field is applied or not; here we will use the second state to refer to the anisotropic microstructure without a magnetic field (i.e. we apply a magnetic field to MRG to create an anisotropic structure and then turn off the magnetic field) [38]. In the third state, a chain-like anisotropic microstructure forms instantaneously when exposed to an external magnetic field [37]. The first and second particle distribution states can exist in an MRG without a magnetic field, though not simultaneously; after applying a magnetic field, a new chain-like structure aligned with the direction of the magnetic field will form. The main difference between the second and third distribution states is that the microstructure of the third state can be recovered after applying an external mechanical stimulus, 
10

11 12

13

14

15

16

17

but the microstructure of the second state is easily destroyed.

Table 1 Relevant characteristic parameters of MRGs with different particle concentrations and different particle distribution states.

\begin{tabular}{|c|c|c|c|c|c|c|c|c|c|}
\hline & \multicolumn{3}{|c|}{ Relaxation time /s } & \multicolumn{3}{|c|}{ Instantaneous modulus $/ \mathrm{kPa}$} & \multicolumn{3}{|c|}{ Stable modulus $/ \mathrm{kPa}$} \\
\hline & Iso- $0 \mathrm{mT}$ & Ani-0mT & Ani-95.6mT & Iso-0mT & Ani-0mT & Ani-95.6mT & Iso-0 $0 \mathrm{mT}$ & Ani-0mT & Ani-95.6mT \\
\hline MRG-40 & 6.84 & 6.32 & 18.69 & 6.79 & 10.77 & 51.43 & 0.18 & 0.25 & 9.28 \\
\hline MRG-50 & 6.33 & 6.77 & 20.79 & 11.03 & 15.96 & 100.28 & 0.19 & & 5.54 \\
\hline MRG-60 & 6.85 & 6.37 & 21.43 & 16.75 & 33.20 & 126.52 & 0.29 & 0.34 & 27.43 \\
\hline MRG-70 & 6.79 & 9.73 & 21.49 & 60.07 & 61.23 & 296.99 & 0.32 & & 90.68 \\
\hline
\end{tabular}

The parameters related to stress relaxation have different value for MRGs with the different particle distribution states: the instantaneous modulus and the stable modulus of an anisotropic MRG are higher than those of an isotropic MRG in the absence of magnetic field; after applying a $95.6 \mathrm{mT}$ magnetic field, the instantaneous modulus and the stable modulus are greatly increased. For example, the instantaneous modulus of anisotropic MRG-70 with a $95.6 \mathrm{mT}$ magnetic field is 4.85 times larger than that of anisotropic MRG-70 without magnetic/field, and the stable modulus becomes 226.7 times larger. This indicates that the anisotropic structure increases the moduli of the MRG, and a magnetic field reinforces this effect. It can be speculated from the microstructure schematic (Figure 3d) that the chain-like particle aggregates are more restricting of the polymer matrix than the randomly dispersed particles. It is more difficult for the restricted polymer molecules to move and slip under an external loading. Moreover, the particle network (consisting of chain-like particle aggregates) can resist external loading to some extent, strengthening the resistance of the MRG to external loading. By applying an external magnetic field, the strength of particle chains and their restricting effect to the polymer matrix will be greatly improved by the magnetic interactions between adjacent iron particles [10]. This is why the relaxation modulus of MRGs in the presence of a magnetic field is much larger than without.

Next, the influence of particle concentration on the stress relaxation of MRGs will be analyzed by combining the experimental results (Figure 3) with the calculations from the stress relaxation model (Table 1). We mainly consider in this study the relaxation processes induced by the polymer matrix and the magneto-mechanical coupling effect between the particle and the matrix. We ignore the time required for the magnetic interaction as it is less important than the relaxation time of polymer matrix. The relaxation time calculated by the model is shorter than the time obtained from the experimental results, because we simplify the stress relaxation model when fitting the experimental results (Figure 3 and Table 1). Only one relaxation time is obtained when using the standard linear solid model to simulate the relaxation process of the polymer. This relaxation time is considered to be induced by the slipping of the polymer chains and the free movement of polymer 
molecules after being stretched and then recovering to their entangling state. Actually, the movements of polymer molecular are more complex, relaxation spectra are needed to accurately describe these complex movements. However, in this study we instead concentrate on the interaction mechanism between the magnetic particles and polymer matrix in the MRG, so the relaxation rule of the polymer matrix can be qualitatively understood from the standard linear solid model.

The relaxation time of low-particle-concentration MRGs with different particle distribution states (isotropic and anisotropic) in the absence of a magnetic field is between $6 \mathrm{~s}$ and $7 \mathrm{~s}$ (Table 1), indicating that the particle distribution has very little influence on the time from the deformation of polymer molecules when applying an external loading to recovery to a balanced state. However, if the particle concentration is high enough, the restricting effect to the polymer matrix shows obvious difference for MRG with different particle distribution states, e.g. the relaxation time of anisotropic MRG-70 without a magnetic field is $9.73 \mathrm{~s}$, which is longer than that of isotropic MRG70. The stronger the restricting effect is, the more time is required for the polymer molecules to relax to the balanced state. When applying an external magnetic field, the stiffness of the particle chains changes quickly; the restricting effect of the particle chains to the polymer matrix becomes stronger, and the relaxation process of the polymer matrix is significantly influenced by the stiffness of particle chains. This influence reflects on both the length of relaxation time (the relaxation time increases to about $21 \mathrm{~s}$ ) and the relaxation modulus. We can also see from Table 1 that the relaxation modulus of the MRG with an external magnetic field increases greatly compared with that of the MRG without a magnetic field. Still, the change in the stable modulus of MRG-70 induced by a magnetic field, for example, is $90.28 \mathrm{kPa}$; this is mainly contributed to the particle chains and is relatively stable.

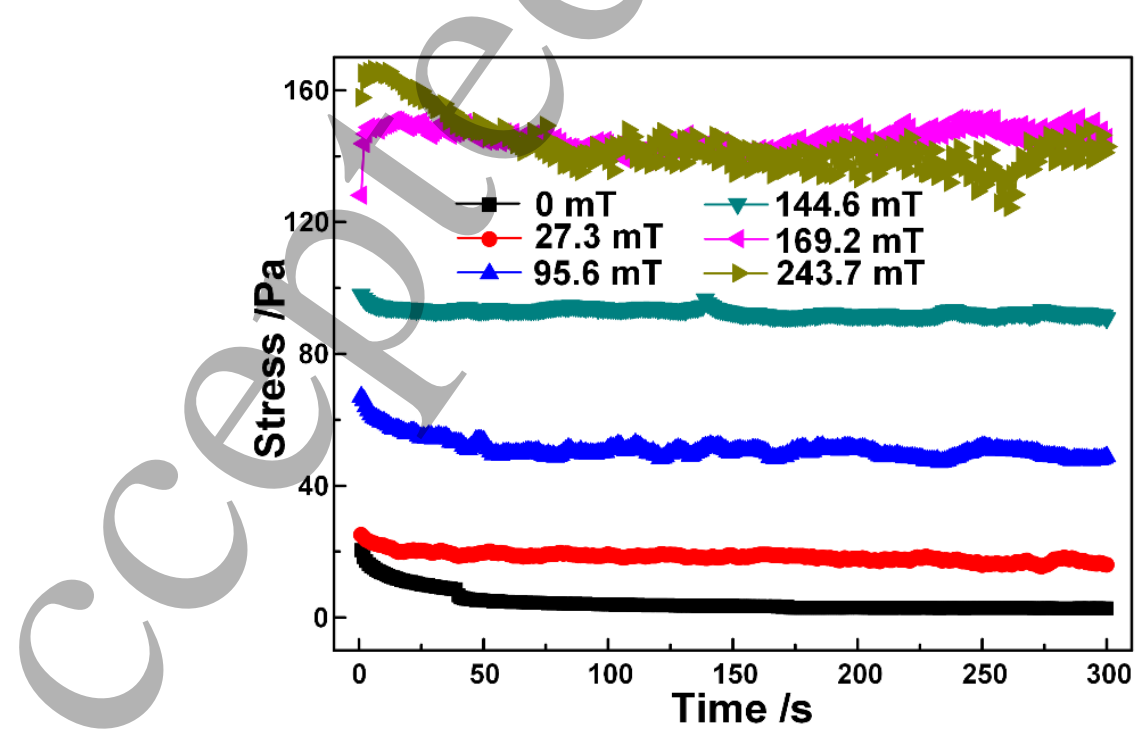

Figure 4 The stress relaxation behaviors of an MR fluid with a particle weight fraction of $70 \%$ under different magnetic fields. 
To confirm this conclusion, we tested the stress relaxation behaviors of an MR fluid with the same particle weight fraction $(70 \mathrm{wt} \%)$ under the same experimental conditions, as shown in Figure 4. The stress relaxation process is not so obvious in the MR fluid. After stepwise shear loading is applied, the MR fluid returns to the balanced state quickly and remains stable, which agrees with the experimental results reported by $\mathrm{Li}$ et al [16]. This result demonstrates that elasticity is dominating in the response of MR fluid to the stepwise shear loading. The gradual increase of the stable stress with an increasing magnetic field suggests that the elasticity, which is sensitive to magnetic field, is mainly caused by the particle chains (the carrier liquid is silicone oil, whose elasticity can be ignored). When applying a magnetic field, the particle chains are the main drivers of change in stable modulus $(90.68 \mathrm{kPa})$, which can be seen by comparing with the stable modulus $(0.40 \mathrm{kPa})$ of anisotropic MRG-70 without a magnetic field. The increment of the instantaneous modulus of anisotropic MRG-70 induced by a $95.6 \mathrm{mT}$ magnetic field is $235.76 \mathrm{kPa}$, much larger than the increment of the stable modulus $(90.28 \mathrm{kPa})$. The difference in the increment for the two kinds of relaxation modulus is $145.48 \mathrm{kPa}$, which is the modulus change of polymer matrix induced by the restricting effect of particle chains to the polymer matrix, i.e. the magneto-mechanical coupling part that we mentioned when discussing the stress relaxation model. It can be seen that the restricting effect of the particle chains to the polymer matrix after applying a magnetic field is very strong, but this will quickly relax over time.

\subsection{Magneto-induced effect}

The magneto-induced effect is key to assessing the performance of magnetorheological materials, and is also the basis for understanding the magneto-mechanical coupling mechanism and assessing its application prospects. The stress relaxation of MRGs under different magnetic fields is shown in Figure 5a, the magnetic field clearly has a strong influence on the stress relaxation of MRGs. To further analyzing the magneto-induced effect, we calculate the characteristic parameters in the stress relaxation model based on the experimental results, as Table 2 shows.
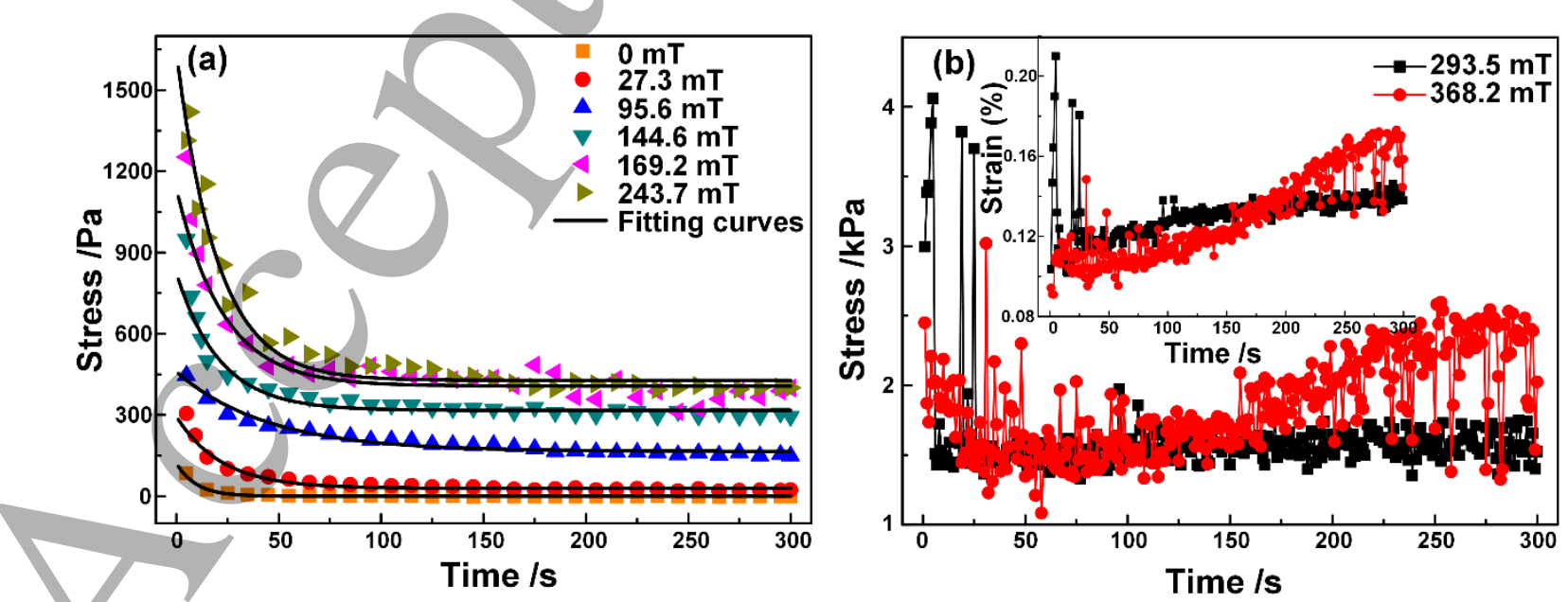
Figure 5 The stress relaxation behaviors of MRG-70 under different magnetic fields (a) and the time dependence of stress under a large magnetic field (b); the inset is the time dependence of the corresponding strain.

Table 2 The relevant characteristic parameters of stress relaxation of MRG-70 under different magnetic fields

\begin{tabular}{c|cccccc}
\hline Magnetic flux density /mT & 0 & 27.3 & 95.6 & 144.6 & 169.2 & 243.7 \\
\hline Relaxation time /s & 9.73 & 21.96 & 21.49 & 21.03 & 22.65 & 19.87 \\
\hline Instantaneous modulus /kPa & 61.23 & 147.72 & 296.99 & 412.58 & 568.81 & 822.19 \\
\hline Stable modulus /kPa & 0.40 & 14.77 & 90.68 & 158.61 & 203.47 & 213.98 \\
\hline
\end{tabular}

Both of the instantaneous modulus and the stable modulus increase gradually with increasing magnetic field strength (Table 2). When applying a $243.7 \mathrm{mT}$ magnetic field, the magneto-induced stable modulus $(213.58 \mathrm{kPa}$, the difference of the modulus with and without magnetic field) is 539 times larger than the stable modulus without magnetic field $(0.40 \mathrm{kPa})$. This great change in the modulus indicates that the MRG presents an obvious magneto-induced effect. Especially at the initial stage of the increasing magnetic field, the variation in the shear modulus is obvious. This result is valuable for some applications that use a moderate magnetic field to greatly adjust the mechanical properties of MRGs. The magneto-dependency of the stress relaxation further confirms that the stress relaxation of MRGs is influenced by the particle chains, the polymer matrix, and the coupling effect between the particle and the matrix. The stiffness of the particle chains and the coupling effect between the particle and the matrix can evidently be enhanced by a magnetic field. The time required to change the particle chain strength is shorter than the relaxation time of the polymer matrix and, as the particle chain can be considered an elastic body, the variation of the relaxation modulus induced by the particle chains is the dominating component of the stable modulus. The increment of the relaxation modulus of the polymer matrix originates from the restriction of particle chains to the polymer matrix (the microstructure schematic of an MRG in the presence of magnetic field is shown in Figure 3d). A strong magnetic field will enhance this restricting effect of particle chains to the polymer matrix, causing the relaxation modulus of polymer matrix to increase. However, change in the shear modulus induced by the restriction to the polymer matrix will disappear gradually with the relaxing of polymer molecules and cannot exist steadily (see the difference between instantaneous modulus and the stable modulus in Table 2).

Unlike the magneto-induced effect on the relaxation modulus, the strength of magnetic field has nearly no influence on the relaxation time of the MRG: the relaxation time in the presence of any magnetic field is approximately $21 \mathrm{~s}$ (see Table 2). However, the difference between relaxation times with and without a magnetic field is large: the relaxation time without a magnetic field is only $9.73 \mathrm{~s}$. This "on-off" phenomenon in the relaxation time demonstrates that there exists a critical value for the restricting effect of the particle chains to the polymer matrix. The restriction will be sufficient if the stiffness of particle chain increases to a critical value. Exceed this value, the increasing particle chain strength will enhance the relaxation modulus of MRG, but the time 
required for the polymer matrix to relax to the equilibrium state will not change substantially. The critical value, corresponding to the critical magnetic field strength, for the sufficient restriction must be determined by carrying out more sophisticated stress relaxation experiment under lower magnetic fields, which is very important for identifying the slipping mechanism of polymer molecules under the restriction of particle chains.

The rotational shear experiment is limited by the static friction between the rotator and the sample. The wall slipping phenomenon will happen if the sample is overexerted by shear loading under a high strain rate or if the shear stress exceeds the static friction due to an increasing shear modulus. It is mentioned in Section 4.1 that the strain amplitude must be achieved quickly to ensure an effective experimental result. If the strain amplitude is set to a large value, the loading rate increases accordingly. As a result, the response stress increases with the high loading rate and may exceed the static friction, causing the wall slipping phenomenon (Figure 2). However, the response stress decreases quickly with time and is lower than the static friction because of the stress relaxation of the MRG, so that the strain will stabilize to the setting value soon after the wall slipping happening. The stress relaxation curves of MRG is effective after the strain reaches the setting value, as shown in Figure 2b. The curves shown in Figure $5 \mathrm{~b}$ are also attributed to the wall slipping, but not as a result of the same slipping mechanism. The MRG becomes much stiffer when a large magnetic field is applied, generating a large response stress. The response stress under a $293.5 \mathrm{mT}$ (or $368.2 \mathrm{mT}$ ) magnetic field exceeds the static friction, again causing the wall slipping phenomenon. As we have mentioned, the relaxation modulus of the MRG under a strong magnetic field will maintain a high value and the stress relaxation is not so pronounced. This means that it is difficult to bring the strain up to the setting value of $0.2 \%$ rather it will fluctuate with time under the setting value (see the inset of Figure 5b). Accordingly, the response stress will fluctuate with the variation of strain, so the stress relaxation result is invalid in this situation. Because the wall slipping mechanism is different, the stress responses of the MRG (Figure $2 b$ and Figure $5 b$ ) are also very different.

\subsection{Temperature-enhanced effect}

The rheological properties of a polymer matrix are usually sensitive to temperature: the matrix becomes softer as temperature rises. Interestingly, a temperature-enhanced effect is found in MRGs under an external magnetic field, i.e. the stable modulus of the MRG increases with the increasing temperature, as shown in Figure 6. The temperature-enhanced effect is also observed in the creep behavior of MRGs [25]. It is found that the temperature-enhanced effect appears only in the presence of a magnetic field, indicating that the magnetic field is the direct reason for the temperature-enhanced effect. However, the rheological performances of MR fluids and MR elastomers deteriorate with a rise in temperature, even under a magnetic field [16, 39-41], 
indicating that variation in the rheological performance of the carrier matrix also plays an important role in the temperature-enhanced effect of MRGs. The inelastic polymer matrix of MRGs is more sensitive to temperature than the silicone oil in MR fluids or the rubber-like matrix of MR elastomers. The polymer matrix of MRGs can change from a solid-like state to a liquid-like state easily within a narrow temperature range $\left(25^{\circ} \mathrm{C} \sim 75^{\circ} \mathrm{C}\right)$ [42]. The polymer matrix in a liquid-like state relaxes easily and the coupling effect between the matrix and the particles weakens greatly in the transition of the polymer matrix from a solid-like to a liquid-like state; thus, the increment of the relaxation modulus decreases as a result of the magneto-mechanical coupling effect between the matrix and the particles. This influence on the modulus is the main contributor to the stress relaxation.

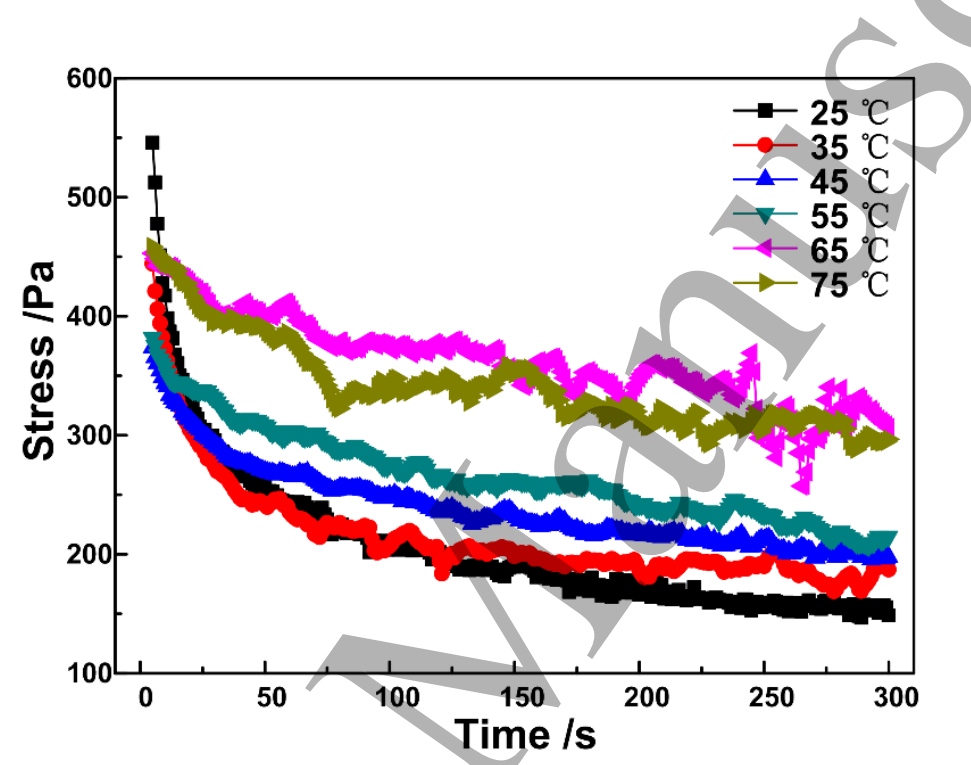

Figure 6 The stress relaxation of MRG-70 under different temperatures, a $95.6 \mathrm{mT}$ magnetic field was applied during all the stress relaxation experiments.

The weakening of the magneto-mechanical coupling effect also makes the particle chains more orderly. This is because the formation of the particle chains under an external magnetic field is a gradually optimized prôcess; it takes time to reach a plateau [43]. The softening of the polymer matrix will accelerate the process of forming stable chain-like structures. An MRG with highly ordered chain-like structures possesses a large shear modulus, resulting in the interesting temperature-enhanced effect. We also notice that the response stress fluctuation becomes more and more obvious with rising temperatures. This phenomenon may be induced by the restructuring process of the particle chains, which confirms the analysis above of the temperature-enhanced effect.

\section{Conclusions and prospects}

The stress relaxation experiment is a simple and effective method for studying the magnetomechanical coupling mechanism in MRGs. The linear viscoelastic range of MRGs is also easily 
determined based on the time dependence of the relaxation modulus under stepwise strain loading with different amplitudes. The stress relaxation of MRGs is found to be significantly influenced by the particle concentration, the particle distribution state, the magnetic field strength, and the temperature. In the absence of a magnetic field, the instantaneous and stable modulus of MRGs with ordered microstructures are larger than those of MRGs whose particles are randomly dispersed. With increasing particle concentration and magnetic field strength, the instantaneous and stable modulus of MRGs will increase accordingly. All of these experimental results are attributed to the magneto-mechanical coupling effect between the particles and the polymer matrix. The relaxation stress originates from the particle chains, the polymer matrix, and the magneto-mechanical coupling between the particles and the polymer matrix. The particle chains are the main contributor to the stable modulus in the presence of a magnetic field. The stiffness of the particle chains can be changed by a magnetic field, but the number of particle chains varies according to the particle concentration. The greater the stiffness or the larger the number of the particle chains, the stronger the restricting effect of the particle chain to the polymer matrix, and the larger the instantaneous modulus of the MRG. Interestingly, the stable relaxation stress increases with a rise in temperature. This temperature-enhanced effect was also found in the previous creep and recovery experiment [25], and is a unique phenomenon in MRGs. Temperature softens the polymer matrix, causing the interaction between the particle and the polymer matrix to weaken, and so the increment of the polymer relaxation modulus induced by the restriction of the particle to the polymer matrix decreases. At the same time, a highly ordered chain-like particle microstructure is more easily formed in a softer polymer matrix, which then enhances the stiffness of the particle chains. Considering all these factors, the relaxation modulus of MRGs also increases with a rise in temperature.

This research helps to verify the magneto-mechanical coupling effect in MRGs. Although the phenomenological model we have established in this work can predict some behavioral tendencies of the key material parameters, i.e. instantaneous modulus, stable modulus, and relaxation time, as they vary with the magnetic field, particle concentration, and temperature, the stress relaxation model is still relatively rough and cannot offer conclusive theoretical support for the experimental results. Next, a theoretical model of MRGs that interprets these experimental results and predicts some magneto-mechanical coupling behaviors based on this work should be explored.

\section{Acknowledgments}

The research reported in this publication was supported by funding from King Abdullah University of Science and Technology (KAUST), the National Natural Science Foundation of China (Grant Nos. 11502256, 11502255, 11602242), and the key project "Computational Solid Mechanics" of the China Academy of Engineering Physics. The authors gratefully thank 
Professor Xinglong Gong at University of Science and Technology of China for his help with experiments and beneficial discussion.

\section{Appendix: the mathematical derivation of the constitutive relation of MRG}

In this paper, Laplace transformation is used to obtain the solution of our proposed model. We start from the right part of Figure $1 \mathrm{~b}$ (the standard linear solid model). According to

$$
\begin{aligned}
& \gamma=\gamma_{m 1}+\gamma_{m 2} \\
& \tau_{m}=G_{m 1} \cdot \gamma_{m 1}=G_{m 2} \cdot \gamma_{m 2}+\eta_{m 2} \cdot \dot{\gamma}_{m 2}
\end{aligned},
$$

we have

$$
\dot{\tau}_{m}+\frac{G_{m 1}+G_{m 2}}{\eta_{m 2}} \tau_{m}=G_{m 1} \dot{\gamma}+\frac{G_{m 1} G_{m 2}}{\eta_{m 2}} \gamma
$$

Assuming that the initial condition is $\begin{aligned} & \tau\left(t \rightarrow 0^{+}\right) \rightarrow 0 \\ & \gamma\left(t \rightarrow 0^{+}\right) \rightarrow 0\end{aligned}$, we define the Laplace transformation as

$$
\begin{aligned}
& L(\dot{\tau})=s \bar{\tau}-\tau(0)=s \bar{\tau} \\
& L(\dot{\gamma})=s \bar{\gamma}-\gamma(0)=s \bar{\gamma}
\end{aligned}
$$

Eq. (A3) is also true for the spring model and the standard linear solid model (the left part and right part of Figure 1b, respectively). Therefore, Eq. (A2) can be changed into

$$
\bar{\tau}_{m}=\frac{s G_{m 1} \eta_{m 2}+G_{m 1} G_{m 2}}{s G_{m 1}+\left(G_{m 1}+G_{m 2}\right)} \bar{\gamma} .
$$

The Laplace transformation of the total shear stress is

$$
\bar{\tau}=\bar{\tau}_{p}+\bar{\tau}_{m}=\left(G\left(H_{0}\right)+\frac{s G_{m 1} \eta_{m 2}+G_{m 1} G_{m 2}}{s \eta_{m 2}+\left(G_{m 1}+G_{m 2}\right)}\right) \bar{\gamma}
$$

If $\gamma$ is a stepwise strain (the stress relaxation model), i.e.

$$
\gamma=\gamma_{0} H(t), H(t)=\left\{\begin{array}{l}
0, t<0 \\
1, t \geq 0
\end{array},\right.
$$

where $H(t)$ is a unit Heaviside function, then

$$
\bar{\gamma}=\frac{\gamma_{0}}{s}
$$

and

$$
\bar{\tau}=\left(G\left(H_{0}\right)+\frac{G_{m 1} G_{m 2}}{G_{m 1}+G_{m 2}}\right) \gamma_{0} \cdot \frac{1}{s}+\frac{G_{m 1}^{2} \gamma_{0}}{G_{m 1}+G_{m 2}} \cdot \frac{1}{s+\frac{G_{m 1}+G_{m 2}}{\eta_{m 2}}}
$$

According to the inverse Laplace transformation formula:

$$
L^{-1}\left(\frac{1}{S}\right)=1(t), L^{-1}\left(\frac{1}{s+\frac{G_{m 1}+G_{m 2}}{\eta_{m 2}}}\right)=e^{-\frac{G_{m 1}+G_{m 2}}{\eta_{m 2}} t},
$$


the total shear stress can be finally obtained, i.e.

$$
\tau=L^{-1}(\bar{\tau})=\gamma_{0}\left(\frac{G_{m 1} G_{m 2}}{G_{m 1}+G_{m 2}}+G\left(H_{0}\right)\right)+\frac{\gamma_{0} G_{m 1}^{2}}{G_{m 1}+G_{m 2}} e^{-\frac{\left(G_{m 1}+G_{m 2}\right) t}{\eta_{m 2}}} .
$$

\section{References}

[1] Hu B, Fuchs A, Huseyin S, Gordaninejad F and Evrensel C 2006 Supramolecular magnetorheological polymer gels J. Appl. Polym. Sci. 100 2464-2479

[2] Wei B, Gong X L, Jiang W Q, Qin L J and Fan Y C 2010 Study on the properties of magnetorheological gel based on polyurethane J. Appl. Polym. Sci. 2010, 118, 2765-2771

[3] Mitsumata T, Wakabayashi T and Okazaki T 2008 Particle dispersibility and giant reduction in dynamic modulus of magnetic gels containing barium ferrite and iron oxide particles J. Phys. Chem. B 112 14132-14139 [4] An H N, Picken S J and Mendes E 2010 Enhanced hardening of soft self-assembled copolymer gels under homogeneous magnetic fields Soft Matter 6: 4497-4503

[5] An H N, Picken S J and Mendes E 2012 Nonlinear rheological study of magneto responsive soft gels Polymer 53 4164-4170

[6] Xuan S H, Zhang Y L, Zhou Y F, Jiang W Q and Gong X L 2012 Magnetic Plasticine (TM): a versatile magnetorheological material J. Mater. Chem. 22 13395-13400

[7] Wu J K, Gong X L, Fan Y C and Xia H S 2011 Physically crosslinked poly(vinyl alcohol) hydrogels with magnetic field controlled modulus Soft Matter 7 6205-6212

[8] Gong X L, Xu Y G, Xuan S H, Guo C Y, Zong, L H and Jiang W Q 2012 The investigation on the nonlinearity of plasticine-like magnetorheological material under oscillatory shear rheometry J. Rheol. 56 1375-1391

[9] An H N, Picken S J and Mendes E 2012 Direct observation of particle rearrangement during cyclic stress hardening of magnetorheological gels Soft Matter 8 11995-12001

[10] Xu Y G, Gong X L, Xuan S H, Zhang W and Fan Y C 2011 A high-performance magnetorheological material: preparation, characterization and magnetic-mechanic coupling properties Soft Matter 7 5246-5254

[11] Shahrivar K and De Vicente J 2013 Thermoresponsive polymer-based magneto-rheological (MR) composites as a bridge between MR fluids and MR elastomers Soft Matter 9 11451-11456

[12] Lakes R 2009 Viscoelastic materials New York: Cambridge University Press

[13] He P S 2013 The structures and properties of polymer Alpha Science Intl. Ltd

[14] An J S, Kwon S H, Choi H J, Jung J H and Kim Y G 2017 Modified silane-coated carbonyl iron/natural rubber composite elastomer and its magnetorheological performance Composite Struct. 160 1020-1026

[15] Ortiz C, Ober C K and Kramer E J 1998 Stress relaxation of a main-chain, smectic, polydomain liquid crystalline elastomer Polymer 39 3713-3718

[16] Li W H, Du H, Chen G, Yeo S H and Guo N Q 2002 Nonlinear rheological behavior of magnetorheological fluids: step-strain experiments. Smart Mater. Struct. 11 209-217

[17] Wang Q, Dong X F, Li L Y, Tong Y and Ou J P 2013 Constitutive description for relaxation behavior of magnetorheological elastomer, Acta Mater. Comp. Sinica 30 138-141

[18] Xu Y G, Gong X L and Xuan S H 2013 Soft magnetorheological polymer gels with controllable rheological properties Smart Mater. Struct. 22075029

[19] Ihlemann J and Besdo D 2003 A phenomenological constitutive model for rubberlike materials and its numerical applications Inter. J. Plast.19 1019-1036

[20] Li W H, Zhou Y and Tian T F 2010 Viscoelastic properties of MR elastomers under harmonic loading Rheol. Acta 49 733-740

[21] Chen L and Jerrams S 2011 A rheological model of the dynamic behavior of magnetorheological elastomers J.Appl. Phys. 110013513 
[22] Liao G J, Gong X L and Xuan S H 2013 Magnetic field-induced compressive property of magnetorheological elastomer under high strain rate Ind. Eng. Chem. Res. 52 8445-8453

[23] Shen Y, Golnaraghi M F and Heppler G R 2004 Experimental research and modeling of magnetorheological elastomers J. Intell. Mater. Syst. Struct. 15 27-35

[24] Jolly M, Carlson J and Munoz B 1996 A model of the behaviour of magnetorheological materials Smart Mater. Struct. 5 607-614

[25] Xu Y G, Gong X L, Xuan S H, Li X F, Qin L J and Jiang W Q 2012 Creep and recovery behaviors of magnetorheological plastomer and its magnetic-dependent properties Soft Matter 8 8483-8492

[26] Lubrda V A, Benson D J and Meyers M A 2003 Strain-rate effects in rheological models of inelastic response Inter. J. Plast. 19 1097-1118

[27] Mckinley G H and Ng T S K 2008 Power law gels at finite strains: the nonlinear rheology of gluten gels $J$. Rheol. 52 417-449

[28] Hough L A, Islam M F, Janmey P A and Yodh A G 2004 Viscoelasticity of single wall carbon nanotube suspensions Phys. Rev. Lett. 93168102

[29] Kobelev V and Schweizer K S 2005 Nonlinear elasticity and yielding of depletion gels J. Chem. Phys. 123 164902

[30] Tong Z, Sun W X, Yang Y R, Wang T, Liu X X and Wang C Y 2011 Large/amplitude oscillatory shear rheology for nonlinear viscoelasticity in hectorite suspensions containing poly(ethylene glycol) Polymer 52 14021409

[31] Wilhelm M, Reinheimer P and Ortseifer M 1999 High sensítivity Fourier-transform rheology Rheol. Acta 38 349-356

[32] Mitsumata T, Honda A, Kanazawa H and Kawai M 2012 Magnetically tunable elasticity for magnetic hydrogels consisting of carrageenan and carbonyl iron particles J. Phys. Chem. B 2012, 116: 12341-12348.

[33] An H N, Picken S J and Mendes E 2012 Nonlinear rheological study of magneto responsive soft gels Polymer 53 4164-4170

[34] Xu Y G, Gong X L, Liu T X and Xuan S H 2013 Magneto-induced microstructure characterization of magnetorheological plastomers using impedance spectroscopy Soft Matter $\mathbf{9}$ 7701-7709

[35] Yu M, Ju B X,Fu J, Liu S Z and Choi S B 2014 The magnetoresistance characteristics of magnetorheological gel under a magnetic field Ind. Eng. Chem. Res. 53 4704-4710

[36] Bergstrom J S and Boyce M C 1999 Mechanical behavior of particle filled elastomers. Rubber Chem. Tech. 72 633-656

[37] De Vicente J, Klingenberg D J and Hidalgo-Alvarez R 2011 Magnetorheological fluids: a review Soft Matter 7 3701-3710

[38] Chen L, Gong X L and Li W H 2007 Microstructures and viscoelastic properties of anisotropic magnetorheological elastomers. Smart Mater. Struct.16 2645-2650

[39] Gordaninejad F and Breese D G 1999 Heating of magnetorheological fluid dampers J. Intell. Mater. Syst. Struct. 10 634-645

[40] Zschunke F, Rivas R and Brunn P O 2005 Temperature behavior of magnetorheological fluids Appl. Rheol. 15 116-121

[41] Zhang W, Gong X L, Xuan S H and Jiang W Q 2011 Temperature-dependent mechanical properties and model of magnetorheological elastomers. Ind. Eng. Chem. Res. 50 6704-6712

[42] Gong X L, Fan Y C, Xuan S H, Xu Y G and Peng C 2012 Control of the damping properties of magnetorheological elastomers by using polycaprolactone as a temperature-controlling component Ind. Eng.

Chem. Res. 51 6395-6403

[43] Liu T X, Xu Y G, Gong X L, Pang H M and Xuan S H 2013 Magneto-induced normal stress of 
2 magnetorheological plastomer. AIP Adv. 3082122 